\title{
Creating a Composite Road Safety Performance Index by a Hierarchical Fuzzy TOPSIS Approach
}

\author{
Qiong Bao ${ }^{1}$, Da Ruan ${ }^{1,2}$, Yongjun Shen ${ }^{1}$, Elke Hermans ${ }^{1}$ \\ 1. Transportation Research Institute (IMOB) \\ Hasselt University \\ Wetenschapspark 1 bus 12, 3590 Diepenbeek, Belgium \\ qiong.bao@student.uhasselt.be \\ \{da.ruan, yongjun.shen, elke.hermans\}@uhasselt.be \\ 2. Belgian Nuclear Research Centre (SCK·CEN) \\ Boeretang 200, $2400 \mathrm{Mol}$, Belgium \\ druan@sckcen.be
}

\begin{abstract}
With the increasing public awareness of the complexity of road safety phenomenon, much more detailed aspects of crash and injury causation rather than only crash data (e.g., the number of road fatalities) are extensively investigated in the current road safety research. Safety performance indicators (SPIs), which are causally related to the number of crashes or to the injury consequences of a crash, are thus rapidly developed and increasingly used. Furthermore, to measure the multidimensional concept of road safety which cannot be captured by a single indicator, the exploration of a composite road safety performance index is attractive and desirable. This study proposes a hierarchical fuzzy TOPSIS method to combine the multilayer SPIs into one overall index by incorporating experts' opinions. Using the number of road fatalities per million inhabitants as a relevant point of reference, the proposed method has proven valuable as an alternative way in creating a composite road safety performance index for a set of European countries. Meanwhile, it effectively handles experts' linguistic expressions instead of crisp values and takes the layered hierarchy of the indicators into account which is seldom considered in the current index research.
\end{abstract}

Keywords-road safety performance indicators; composite index; multi-criteria decision making; TOPSIS; fuzzy set theory; hierarchical structure

\section{INTRODUCTION}

As one of the most fast growing sectors, transport system is expected to experience an accelerated expansion in the near future. However, rapid growth of traffic volume, especially the motorized road mobility, has also resulted in continuously increasing safety problems. Worldwide, an estimated 1.2 million people are killed in road crashes each year, and as many as 50 million more are injured [1]. It not only imposes huge economic costs representing between 1 and 3 percent of GDP in most countries, but also causes great emotional and financial stress to the millions of families affected. More seriously, projections indicate that these figures will increase by about $65 \%$ over the next 20 years unless there is new commitment to prevention [1].
Given the high number of road casualties and the corresponding suffering and socio-economic costs, measures are urgently needed to reduce this number and make progress in road safety. In this respect, safety performance indicators (SPIs), which are causally related to the number of crashes or to the injury consequences of a crash (e.g., seat belt wearing rate), are rapidly developed and increasingly used, especially over the last decade (e.g., [2-4]). Knowledge on these indicators is valuable in understanding the processes that lead to crashes, determining the main risk factors, identifying the corresponding interventions, and monitoring the effectiveness of the safety actions that are taken.

However, various underlying risk factors of road safety exist, and each risk factor (e.g., protective system) could be possibly represented by several appropriate SPIs (e.g., seat belt wearing rate in front and rear seats, respectively) constituting a layered hierarchy. Thus, a simple comparison per indicator only shows a small piece of the road safety picture, and it can be misleading since different countries may operate in different circumstances with different focal points. Consequently, to measure the multi-dimensional concept of road safety which cannot be captured by a single indicator, the exploration of a composite road safety index is attractive and desirable. The index thus presents the overall road safety picture by capturing a multitude of risk information in one index score, and offers advantages in terms of communication, benchmarking, and prioritizing road safety actions [5,6].

Compared to other fields such as environment, economy, and society, the development of a composite index for road safety is relatively new. This is because the traditional research mainly focuses on the road safety final outcomes in terms of fatalities per head of population, vehicle fleet or other measures of exposure [7]. However, they are limited to the "worst case scenario" in the unsafe operational conditions of traffic system, and are insufficient in explaining more detailed aspects of crash causation and injury prevention. Recent studies (e.g., [5,8-10]) have been carried out for the development of a composite road safety index, in which both objective weighting methods (e.g., principal component analysis, factor analysis, data 
envelopment analysis, neural networks and rough set theory) and subjective weighting methods (e.g., analytical hierarchy process and budget allocation) were adopted. However, there are still some limitations in practice that need to be paid attention to. Firstly, relatively small number of basic indicators was considered, for example, in [9] and [10], only one quantitative indicator was selected for each risk factor, which might be insufficient in reflecting the entire situation of the risk factors. While in [5] and [8], although one or several indicators were suggested for each factor, all of them were treated to be in the same layer, and the information on the layered hierarchy was ignored. Secondly, of all the weighting methods mentioned above, those objective ones rely mostly on the quality of information about the indicators. In other words, they are usually used with the precondition that all the indicators are measurable and quantitative. If some of them are specified with either ordinal measures or the help of expert subjective judgments, these methods may not be applied directly. Moreover, concerning those subjective weighting methods based on experts' opinions, it is known that experts prefer to give linguistic valued assessments rather than crisp value judgments, such as 'low', 'relatively low', and 'high', 'extremely high'. This phenomenon results from inability to explicitly state their preferences due to the fuzzy nature of the comparison process. In this case, precise mathematical approaches are not enough to tackle such uncertain variables and derive a satisfactory solution [11].

In this study, we investigate the Technique for Order Preference by Similarity to Ideal Solution (TOPSIS) [12], which is one of the well-known classical multi-criteria decision making (MCDM) methods. To deal with the above-mentioned linguistic expression given by experts and the layered hierarchy of the indicators, we propose a hierarchical fuzzy TOPSIS model to combine the multilayer SPIs into one overall index by incorporating experts' opinions. Using the number of road fatalities per million inhabitants as a relevant point of reference, the proposed method has proven valuable as an alternative way in creating a composite road safety performance index for a set of European countries.

\section{SAFETY PERFORMANCE INDICATORS}

Based on a review of safety policies in the European Union and its member states, a number of road safety risk factors were designated as central to road safety activities in Europe and were selected for the development of SPIs [2,6]. They are: alcohol and drugs; speed; protective systems; vehicle; roads; and trauma management. Moreover, each risk factor needs to be measured by at least one performance indicator which is policy relevance, data availability and reliability. In this study, we construct a hierarchical structure of SPIs as in Fig. 1. More specifically, for alcohol and drugs, the percentage of drivers disrespecting the alcohol limit is the indicator (A1); the speed indicator is the percentage of drivers exceeding the speed limit in built-up areas (S1); the protective systems are represented by the seat belt wearing rate in front and rear seats, respectively (P1 and P2); the age distribution and the composition of the vehicle fleet are the two main aspects reflecting the vehicle performance, and totally four indicators are selected, which are the share of passenger cars of maximum five years old (V1), the median age of the passenger car fleet (V2), the share of motorcycles and heavy goods vehicles (HGV) in the vehicle fleet, respectively (V3 and V4); the motorways density (R1) and the share of motorways in total road length (R2) describe the roads domain, and for trauma management the health expenditure as share of the gross domestic product (GDP) is the selected indicator (T1).

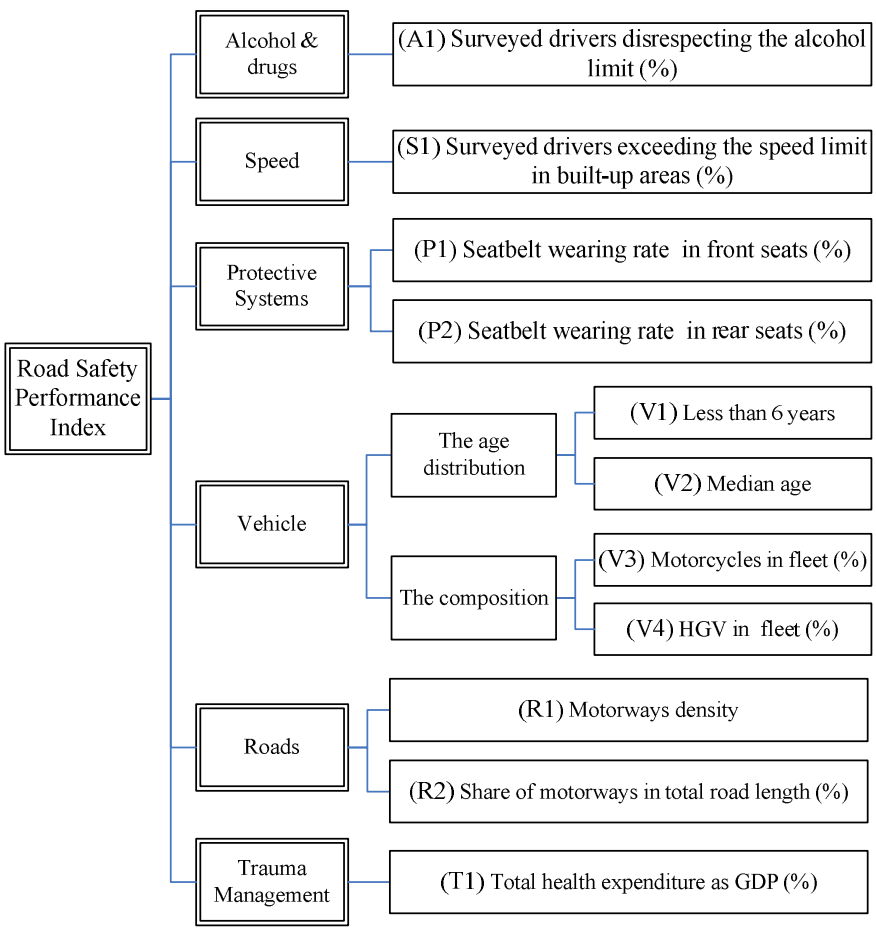

Figure 1. The hierarchical structure of the SPIs

From a wide range of international databases and recent publications of international working groups [13-16], values related to 2003 are obtained for the developed 11 SPIs of 21 European countries being Austria (AT), Belgium (BE), Cyprus (CY), Czech Republic (CZ), Denmark (DK), Estonia (EE), Finland (FI), France (FR), Germany (DE), Greece (EL), Hungary (HU), Ireland (IE), Italy (IT), the Netherlands (NL), Poland (PL), Portugal (PT), Slovenia (SL), Spain (ES), Sweden (SE), Switzerland $(\mathrm{CH})$, and United Kingdom (UK). In the following sections, the above 11 SPIs are combined into an overall road safety performance index for each country by using the hierarchical fuzzy TOPSIS method, and the 2003 number of road fatalities per million inhabitants for these 21 countries will serve as the reference point of the index results.

\section{THE HIERACHICAL FUZZY TOPSIS}

Hwang and Yoon in 1981 developed TOPSIS as one of the well known classical MCDM methods. It bases upon the concept that the chosen alternative should have the shortest distance from the positive-ideal solution (PIS) and the farthest distance from the negative-ideal solution (NIS), in which the PIS is formed as a composite of the best performance values exhibited by any alternative for each criterion, and the NIS is the composite of the worst performance values. Proximity to each of these performance poles is measured in the Euclidean 
sense (e.g., square root of the sum of the squared distances along each axis in the 'criterion space'), with optional weighting of each criterion. Since the construction process of this method is transparent which makes it easily understood by the general public, and can be used to support a desired policy, during the last three decades, a large amount of literature exists involving TOPSIS theory and applications [17].

However, due to the ever increasing complexity of today's performance evaluation and decision making activities, the application of the classical TOPSIS methods may face serious practical problems from the criteria (which usually construct a hierarchical structure) to the weights (which perhaps contain imprecision or vagueness inherent in the information such as linguistic expression instead of crisp values given by experts). Therefore, we propose a hierarchical fuzzy TOPSIS method as a nature extension of the classical TOPSIS. The main procedure of this method has in the following ten steps:

\section{Step 1 Identify a decision matrix}

To obtain the performance of a set of alternatives on a given set of criteria, a decision matrix, $\widetilde{\mathbf{D}}$, of $m \times n$ dimension is constructed firstly. Consider a two-layer situation of $m$ alternatives $A_{i}(i=1,2, \ldots, m)$, each including $n$ main criteria $(M C)$, and $r$ sub-criteria $(S C)$. Assume each main criterion has $r_{j}$ sub-criteria, thereby the total number of sub-criteria $r$ is equal to the sum of $r_{j}(j=1,2, \ldots, n) . \tilde{x}_{i j k}$ represents the value of the $k$ th sub-criteria within the $j$ th main criteria of the $i$ th alternative, which can be crisp data or linguistic variables (e.g., low, medium, and high), and can be further represented by fuzzy numbers, e.g., $\tilde{x}_{i j k}=\left(a_{i j k}, b_{i j k}, c_{i j k}\right)$. Thus, a hierarchical fuzzy multi-criteria decision-making problem can be concisely expressed in matrix format as in (1):

$$
\begin{aligned}
& \begin{array}{cccc}
M C_{1} & M C_{2} & \cdots \cdots & M C_{n}
\end{array}
\end{aligned}
$$

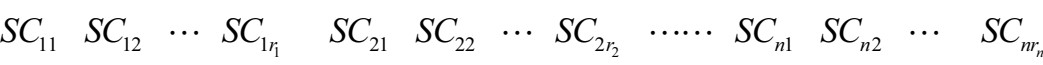

$$
\begin{aligned}
& \tilde{\mathbf{D}}=\begin{array}{c}
A_{1} \\
A_{2} \\
\vdots \\
A_{m}
\end{array}\left[\begin{array}{ccccccccccccc}
\tilde{x}_{111} & \tilde{x}_{112} & \cdots & \tilde{x}_{1 r_{1}} & \tilde{x}_{121} & \tilde{x}_{122} & \cdots & \tilde{x}_{12 r_{2}} & \cdots \cdots & \tilde{x}_{1 n 1} & \tilde{x}_{1 n 2} & \cdots & \tilde{x}_{1 n r_{n}} \\
\vdots & \tilde{x}_{212} & \cdots & \tilde{x}_{21 r_{1}} & \tilde{x}_{221} & \tilde{x}_{222} & \cdots & \tilde{x}_{22 r_{2}} & \cdots \cdots & \tilde{x}_{2 n 1} & \tilde{x}_{2 n 2} & \cdots & \tilde{x}_{2 n r_{n}} \\
\tilde{x}_{m 11} & \tilde{x}_{m 12} & \cdots & \tilde{x}_{m 1 r_{1}} & \tilde{x}_{m 21} & \tilde{x}_{m 22} & \cdots & \tilde{x}_{m 2 r_{2}} & \cdots \cdots & \tilde{x}_{m n 1} & \tilde{x}_{m n 2} & \cdots & \tilde{x}_{m n n_{n}}
\end{array}\right]
\end{aligned}
$$

Step 2 Normalize the decision matrix

All the elements in the decision matrix must be normalized to the same units, so that all possible criteria in the decision problem can be considered simultaneously. Here, convert the decision making matrix $(\widetilde{\mathbf{D}})$ to a dimensionless matrix $\left(\widetilde{\mathbf{D}}^{\prime}\right)$ is done by using linear scale transformation shown in (2).

$\tilde{r}_{i j k}=\left\{\begin{array}{l}\tilde{x}_{i j k}(:) \tilde{x}_{j k}^{*}=\left(\frac{a_{i j k}}{c_{j k}^{*}}, \frac{b_{i j k}}{b_{j k}^{*}}, \frac{c_{i j k}}{a_{j k}^{*}}\right), \forall_{j k}, \tilde{x}_{j k} \text { is a benefit criterion } \\ \tilde{x}_{j k}^{-}(:) \tilde{x}_{i j k}=\left(\frac{a_{j k}^{-}}{c_{i j k}}, \frac{b_{j k}^{-}}{b_{i j k}}, \frac{c_{j k}^{-}}{a_{i j k}}\right), \forall_{j k}, \tilde{x}_{j k} \text { is a cost criterion }\end{array}\right.$

where $\tilde{r}_{i j k}$ are normalized values. $\tilde{x}_{j k}^{*}=\left(a_{j k}^{*}, b_{j k}^{*}, c_{j k}^{*}\right)$ and $\tilde{x}_{j k}^{-}=\left(a_{j k}^{-}, b_{j k}^{-}, c_{j k}^{-}\right)$present the largest and the lowest value of each sub-criterion, respectively.

Step 3 Determine the main criteria weight matrix $\tilde{w}_{M C}$

Determination of the relative importance of each criterion is critical and several approaches exist. In this study, the geometric mean method is used to combine different individual weights given by the group decision makers into a single collective weight for each corresponding criterion.

$$
\left.\begin{array}{r|c}
M C_{1} & \tilde{w}_{1} \\
M C_{2} & \tilde{w}_{2} \\
\vdots & \vdots \\
\tilde{w}_{M C}= & \tilde{w}_{j} \\
\vdots & \vdots \\
M C_{n} & \tilde{w}_{n}
\end{array}\right]
$$

where $\tilde{w}_{j}(j=1,2, \ldots, n)$ is the geometric mean of the main criteria weight scores assigned by the $K$ experts, which is calculated according to (4).

$$
\tilde{w}_{j}=\sqrt[K]{\tilde{w}_{j}^{1}(\cdot) \tilde{w}_{j}^{2}(\cdot) \cdots(\cdot) \tilde{w}_{j}^{K}}
$$

where $\tilde{w}_{j}^{K}$ is the weight score assigned by the $K$ th decision maker.

The weights $\tilde{w}_{j}$ are also linguistic variables (e.g., important and unimportant), and can be represented by fuzzy numbers, e.g., $\tilde{w}_{j}=\left(w_{j 1}, w_{j 2}, w_{j 3}\right)$.

Step 4 Obtain the sub-criteria weight matrix $\tilde{w}_{S C}$ concerning the corresponding main criteria

Based on the same method, the importance weight scores of the sub-criteria with respect to the corresponding main criteria are presented in (5). 


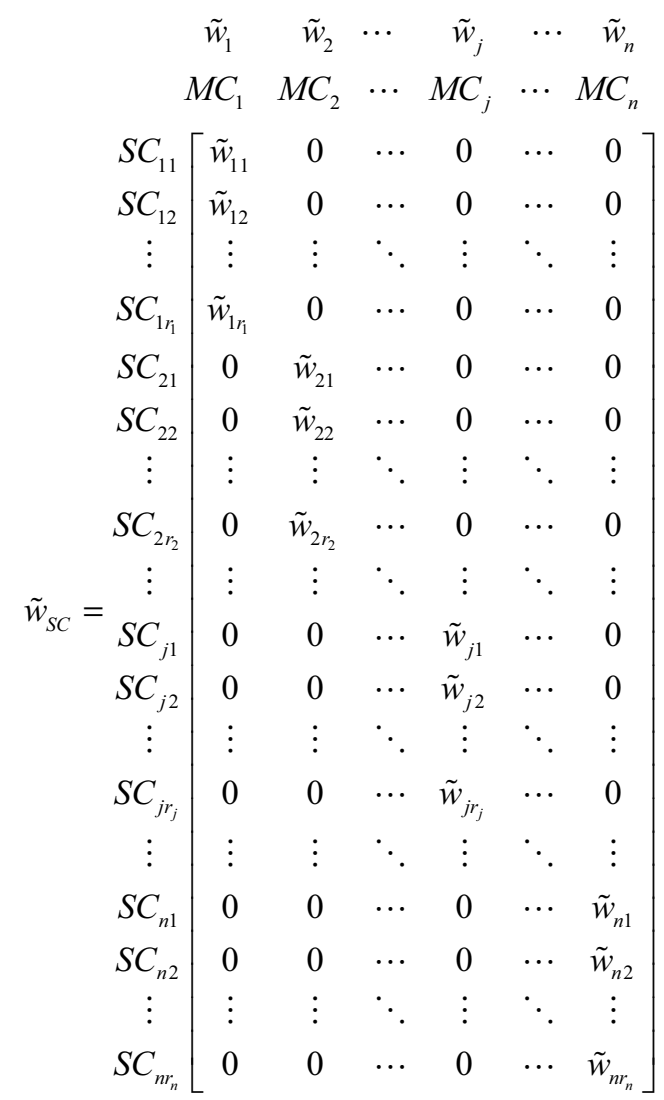

where $\tilde{w}_{j r_{j}}(j=1,2, \ldots, n)$ is the geometric mean of the subcriteria weight scores with respect to the corresponding main criteria given by the $K$ experts.
Step 5 Calculate the final weight score for each subcriterion

The final weight score for each sub-criterion is the product of the main criterion weight score and the sub-criterion weight score with respect to the corresponding main criterion as shown in (6).

$\tilde{W}_{S C j}=\tilde{w}_{M C j}(\cdot) \tilde{w}_{S C j}=\tilde{w}_{j}(\cdot)\left[\begin{array}{c}\tilde{w}_{j 1} \\ \tilde{w}_{j 2} \\ \vdots \\ \tilde{w}_{j r_{j}}\end{array}\right]=\left[\begin{array}{c}\tilde{w}_{S C j 1} \\ \tilde{w}_{S C j 2} \\ \vdots \\ \tilde{w}_{S C j r_{j}}\end{array}\right],(j=1,2, \ldots, n)$

where $\tilde{W}_{S C j}$ represents the final sub-criteria weight matrix concerning the $j$ th main criterion. $\tilde{w}_{M C j}$ and $\tilde{w}_{S C j}$ denote the $j$ th main criterion weight score and the sub-criteria weight scores with respect to this main criterion, respectively.

Step 6 Compute the weighted normalized decision matrix

By multiplying the fuzzy criteria values and the final fuzzy weight scores of each sub-criterion, the weighted normalized fuzzy decision matrix $\tilde{\mathbf{V}}$ is achieved as calculated by (7).

$$
\tilde{v}_{i j k}=\tilde{r}_{i j k}(\cdot) \tilde{w}_{S C j}, \forall{ }_{j}, j
$$

The result of (7) can be summarized as (8).

$$
\begin{aligned}
& \begin{array}{cccc}
M C_{1} & M C_{2} & \cdots \cdots & M C_{n}
\end{array} \\
& \begin{array}{lllllllllllll}
S C_{11} & S C_{12} & \cdots & S C_{1 r_{1}} & S C_{21} & S C_{22} & \cdots & S C_{2 r_{2}} & \cdots \cdots & S C_{n 1} & S C_{n 2} & \cdots & S C_{n r_{n}}
\end{array}
\end{aligned}
$$

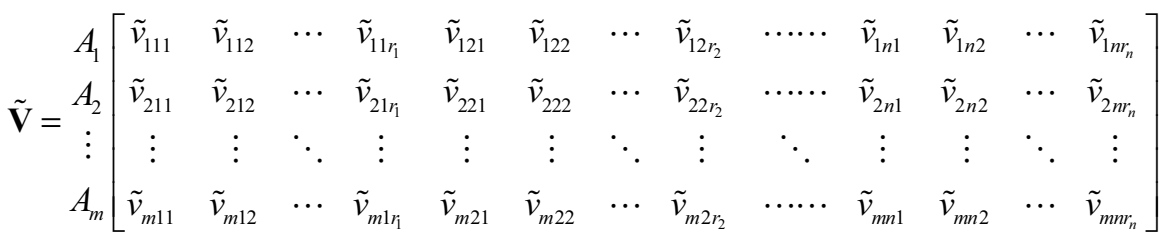

Subsequently, the fuzzy addition principle is used to aggregate the values within each main criterion as follows.

$$
\tilde{v}_{i j}^{\prime}=\sum_{k=1}^{r_{j}} \tilde{v}_{i j k}, j=1,2, \ldots, n .
$$

The matrix $\tilde{\mathbf{V}}$ is thus converted into $\tilde{\mathbf{V}}^{\prime}$ :

$$
\begin{aligned}
& \begin{array}{llll}
M C_{1} & M C_{2} & \cdots & M C_{n}
\end{array} \\
& \tilde{\mathbf{V}}^{\prime}=\begin{array}{c}
A_{1} \\
A_{2} \\
\vdots \\
A_{m}
\end{array}\left[\begin{array}{cccc}
\tilde{v}_{11}^{\prime} & \tilde{v}_{12}^{\prime} & \cdots & \tilde{v}_{1 n}^{\prime} \\
\tilde{v}_{21}^{\prime} & \tilde{v}_{22}^{\prime} & \cdots & \tilde{v}_{2 n}^{\prime} \\
\vdots & \vdots & \ddots & \vdots \\
\tilde{v}_{m 1}^{\prime} & \tilde{v}_{m 2}^{\prime} & \cdots & \tilde{v}_{m n}^{\prime}
\end{array}\right]
\end{aligned}
$$

Step 7 Identify the fuzzy positive ideal solution, $\tilde{A}^{*}$, and the fuzzy negative ideal solution, $\tilde{A}^{-}$.

We now define the fuzzy positive ideal solution (FPIS), i.e., $\tilde{A}^{*}=\left[\tilde{v}_{1}^{*}, \ldots, \tilde{v}_{n}^{*}\right]$ and fuzzy negative ideal solution (FNIS), i.e., $\tilde{A}^{-}=\left[\tilde{v}_{1}^{-}, \ldots, \tilde{v}_{n}^{-}\right]$. The $\tilde{v}_{j}^{*}$ and $\tilde{v}_{j}^{-}$are the fuzzy numbers with the largest and the smallest generalized mean, respectively. The generalized mean for fuzzy number $\tilde{v}_{i j}=\left(a_{i j}, b_{i j}, c_{i j}\right), \forall j$, is defined as:

$$
M\left(\tilde{v}_{i j}\right)=\frac{-a_{i j}^{2}+c_{i j}^{2}-a_{i j} b_{i j}+b_{i j} c_{i j}}{\left[3\left(-a_{i j}+c_{i j}\right)\right]}
$$


For each column $j$, we find the greatest generalized mean as $\tilde{v}_{j}^{*}$ and the lowest generalized mean as $\tilde{v}_{j}^{-}$. Consequently, the FPIS ( $\left.\tilde{A}^{*}\right)$ and FNIS $\left(\tilde{A}^{-}\right)$are derived.

Step 8 Obtain the separation measures $\tilde{S}_{i}^{*}$ and $\tilde{S}_{i}^{-}$

The separation measures are defined as:

$$
\begin{aligned}
& \tilde{S}_{i}^{*}=\sum_{j=1}^{n} \tilde{D}_{i j}^{*}, \quad i=1, \ldots, n \\
& \tilde{S}_{i}^{-}=\sum_{j=1}^{n} \tilde{D}_{i j}^{-}, \quad i=1, \ldots, n
\end{aligned}
$$

where the difference Euclidean distances $\tilde{D}_{i j}^{*}$ and $\tilde{D}_{i j}^{-}$are given as follows:

$$
\begin{gathered}
\tilde{D}_{i j}^{*}=\left\{\begin{array}{lll}
1-\frac{c_{i j}-a^{*}}{b^{*}+c_{i j}-a^{*}-b_{i j}} & \text { for } b_{i j}<b^{*} \\
1-\frac{c^{*}-a_{i j}}{b_{i j}+c^{*}-a_{i j}-b^{*}} & \text { for } b^{*}<b_{i j} &
\end{array} \quad \forall i, j\right.
\end{gathered}
$$

where $\tilde{v}_{i j}=\left(a_{i j}, b_{i j}, c_{i j}\right)$ represents arbitrary element of matrix $\tilde{\mathbf{V}}^{\prime} ; \tilde{v}_{j}^{*}=\left(a^{*}, b^{*}, c^{*}\right)$ and $\tilde{v}_{j}^{-}=\left(a^{-}, b^{-}, c^{-}\right)$are the fuzzy numbers with the largest generalized mean and the smallest generalized mean, respectively.

Note that both $\tilde{D}_{i j}^{*}$ and $\tilde{D}_{i j}^{-}$are crisp values now. Therefore, the separation measures $\tilde{S}_{i}^{*}$ and $\tilde{S}_{i}^{-}$can be calculated according to (12) and (13).

Step 9 Compute the relative closeness to ideals

The relative closeness index is used to combine $\tilde{S}_{i}^{*}$ and $\tilde{S}_{i}^{-}$indices obtained in Step 8, which is calculated as follows:

$$
C_{i}=S_{i}^{-} /\left(S_{i}^{*}+S_{i}^{-}\right)
$$

\section{Step 10 Prioritize Alternatives}

According to the composite index value $C_{i}$, the set of alternatives can be ranked from the most preferred to the least preferred feasible solutions. $C_{i}$ is also called the overall or composite index score of alternative $A_{i}$.

\section{APPLICATION AND DISCUSSIONS}

In this section, we apply the proposed hierarchical fuzzy TOPSIS method to combine the 11 layered SPIs (see Fig. 1) into an overall road safety performance index for the 21
European countries. The indicator values are all crisp in this study, and they constitute a hierarchical decision making matrix, $\tilde{\mathbf{D}}$, whereas the weight scores for the main risk factors and the (sub-)indicators are given by eight road safety experts with linguistic variables.

According to the hierarchical fuzzy TOPSIS method described in Section III, the hierarchical decision making matrix need to be normalized and converted into a dimensionless one, which is $\tilde{\mathbf{D}}^{\prime}$. In this case, 5 out of 11 SPIs, (i.e., the percentage of surveyed car drivers disrespecting the alcohol limit (A1); the percentage of surveyed car drivers exceeding the speed limit in built-up areas (S1); the median age of the passenger car fleet (V2); the share of motorcycles in the vehicle fleet (V3) and the share of HGV in the vehicle fleet (V4)) are the cost indicators, while the remaining six are benefit ones. After normalization, all the indicator values in the decision matrix $\tilde{\mathbf{D}}^{\prime}$ are expected to be the higher the better.

Moreover, based on the triangular membership function, the weight scores expressed by linguistic terms are transferred into positive triangular fuzzy numbers as in Table I.

TABLE I. TRIANGULAR FUZZY NUMBERS FOR THE LINGUISTIC TERMS OF INDICATOR WEIGHTS

\begin{tabular}{|c|c|}
\hline Linguistic terms & Triangular fuzzy numbers \\
\hline Absolutely unimportant & $(0,0,1 / 6)$ \\
\hline Unimportant & $(0,1 / 6,2 / 6)$ \\
\hline Less important & $(1 / 6,2 / 6,3 / 6)$ \\
\hline Important & $(2 / 6,3 / 6,4 / 6)$ \\
\hline More important & $(3 / 6,4 / 6,5 / 6)$ \\
\hline Strongly important & $(4 / 6,5 / 6,1)$ \\
\hline Absolutely important & $(5 / 6,1,1)$ \\
\hline
\end{tabular}

By calculating the product of each main risk factor weight scores and the (sub-)indicator weight scores with respect to the corresponding risk factor, we obtain the final weight scores for each indicator. Subsequently, the weighted normalized fuzzy decision matrix $\tilde{\mathbf{V}}$ is achieved by computing the product of the normalized hierarchical decision matrix $\tilde{\mathbf{D}}^{\prime}$ and the fuzzy weight scores for each indicator. After aggregating the values belonging to each main risk factor by the fuzzy addition principle, we obtain the final weighted normalized fuzzy decision matrix $\tilde{\mathbf{V}}^{\prime}$.

It should be mentioned here that each element in $\tilde{\mathbf{V}}^{\prime}$ is a fuzzy number, so its generalized mean $M\left(\tilde{v}_{i j}\right)$ should be figured out according to (11). The largest generalized mean and the smallest generalized mean of each indicator could then be picked out constituting the FPIS ( $\left.\tilde{A}^{*}\right)$ and the FNIS $\left(\tilde{A}^{-}\right)$. Now, the Euclidean distances $\tilde{D}_{i j}^{*}$ and $\tilde{D}_{i j}^{-}$are calculated as in (14) and (15), and the separation measures $\tilde{S}_{i}^{*}$ and $\tilde{S}_{i}^{-}$, as well as the final composite index scores $C_{i}$ are subsequently obtained. The results are presented in Table II, together with the rankings based on the number of road fatalities per million inhabitants. 
TABLE II. THE RESULTS FROM THE HIERARCHICAL FUZZY TOPSIS AND THE NUMBER OF FATALITY PER MILLION INHABITANTS $(F)$

\begin{tabular}{|c|c|c|c|c|}
\hline Country & $\boldsymbol{C}$ & Ranking & $\boldsymbol{F}$ & $\begin{array}{c}\text { Fatality } \\
\text { Ranking }\end{array}$ \\
\hline Austria & 0.544 & 10 & 115 & 11 \\
\hline Belgium & 0.501 & 12 & 117 & 12 \\
\hline Cyprus & 0.297 & 21 & 134 & 17 \\
\hline Czech Republic & 0.463 & 15 & 142 & 18 \\
\hline Denmark & 0.672 & 3 & 80 & 6.5 \\
\hline Estonia & 0.355 & 20 & 121 & 13.5 \\
\hline Finland & 0.540 & 11 & 73 & 4 \\
\hline France & 0.560 & 9 & 101 & 9 \\
\hline Germany & 0.679 & 2 & 80 & 6.5 \\
\hline Greece & 0.396 & 18 & 146 & 19 \\
\hline Hungary & 0.413 & 17 & 131 & 16 \\
\hline Ireland & 0.562 & 8 & 84 & 8 \\
\hline Italy & 0.420 & 16 & 105 & 10 \\
\hline Netherlands & 0.630 & 6 & 63 & 3 \\
\hline Poland & 0.395 & 19 & 149 & 21 \\
\hline Portugal & 0.469 & 14 & 148 & 20 \\
\hline Slovenia & 0.592 & 7 & 121 & 13.5 \\
\hline Spain & 0.499 & 13 & 130 & 15 \\
\hline Sweden & 0.715 & 1 & 59 & 1 \\
\hline Switzerland & 0.653 & 5 & 74 & 5 \\
\hline United Kingdom & 0.654 & 4 & 61 & 2 \\
\hline & & & & \\
\hline & & & \\
\hline
\end{tabular}

The ranking results derived from the hierarchical fuzzy TOPSIS method show that Sweden is the best performing country, which is the same as the fatality ranking. Taking a close look at the two ranking results, we find that over half of the countries have the difference in rankings with no more than two positions, and about two thirds are no more than three positions, which indicates the high similarity between the derived road safety performance index and the final outcome. The Pearson's correlation analysis further reveals that the composite road safety performance index score of the 21 European countries is highly correlated with their number of fatalities per million inhabitants, which is -0.810 .

\section{CONCLUSIONS}

Composite indexes are increasingly recognized as a useful tool in policy analysis and public communication. In this study, we mainly focused on the use of multi-criteria decision making framework for composite index research in the context of road safety. In this respect, a set of hierarchical safety performance indicators was combined into an overall index by applying one of the well-known MCDM techniques, i.e., the TOPSIS method. Meanwhile, to deal with the subjective kind of uncertainty on data (i.e., linguistic variables given by experts) and the hierarchical structure of the indicators, a hierarchical fuzzy TOPSIS method was realized and proved valuable in creating a composite road safety performance index. First, the construction process of this method is transparent which makes it easily understood by the general public, and can be used to support a desired policy. Second, the linguistic valued judgments from experts which are usually expressed by using fuzzy numbers can be easily integrated in the classical TOPSIS. Moreover, the realization of a hierarchical TOPSIS method which enables taking the hierarchical structure of indicators into account makes it particularly suitable in combining layered road safety performance indicators into one index. In the application case, the derived composite index scores shown a relatively high correlation with the number of road fatalities per million inhabitants, which indicates the effectiveness of the proposed hierarchical fuzzy TOPSIS method, and further implies the feasibility of applying this method to a great number of composite index studies in other wide ranging fields.

\section{REFERENCES}

[1] World Health Organization (WHO), "World Report on Road Traffic Injury Prevention," WHO, Geneva, 2004.

[2] European Transport Safety Council (ETSC), "Transport Safety Performance Indicators," ETSC, Brussels, 2001.

[3] M.A. Vis, "State-of-the-art Report on Road Safety Performance Indicators," Deliverable D3.1 of the EU FP6 project SafetyNet, 2005.

[4] Organisation for Economic Cooperation and Development/International Transport Forum (OECD/ITF), "Towards Zero: Ambitious Road Safety Targets and the Safe System Approach," Joint Transport Research Centre of the OECD/ITF, Paris, 2008

[5] F. Wegman, J. Commandeur, E. Doveh, V. Eksler, V. Gitelman, S Hakkert, D. Lynam and S. Oppe, "SUNflowerNext: Towards a Composite Road Safety Performance Index," Deliverable D6.16 of the EU FP6 project SafetyNet, 2008.

[6] E. Hermans, A Methodology for Developing a Composite Road Safety Performance Index for Cross-country Comparison, PhD Thesis, Hasselt University, Hasselt, Belgium, 2009.

[7] E. Hermans, G. Wets and F. Van den Bossche, "Describing the evolution in the number of highway deaths by decomposition in exposure, accident risk, and fatality risk," In Transportation Research Record: Journal of the Transportation Research Board, No. 1950, Transportation Research Board of the National Academies, Washington, D.C., pp. 1-8, 2006.

[8] G. Al Haji, Towards a Road Safety Development Index. Department of Science and Technology, PhD Thesis, Campus Norrköping, Linköping University, Norrköping, Sweden, 2005

[9] E. Hermans, F. Van den Bossche and G. Wets, "Combining road safety information in a performance index.," Accident Analysis and Prevention, Vol. 40 (4), pp. 1337-1344, 2008.

[10] Y. Shen, T. Li, E. Hermans, D. Ruan, G. Wets, K. Vanhoof and T. Brijs, "A hybrid system of neural networks and rough sets for road safety performance indicators," Soft Computing, 2010, in press.

[11] P.M. Pardalos and D. Du, (Eds) , Fuzzy Multi-Criteria Decision Making: Theory and Applications with Recent Developments, Springer, US, 2008.

[12] C.L. Hwang and K. Yoon, Multiple Attribute Decision Making: Methods and Applications, Springer-Verlag, Berlin/Heidelberg/New York, 1981.

[13] SARTRE Consortium, European Drivers and Road Risk. SARTRE 3 reports, 2004.

[14] European Transport Safety Council (ETSC), Increasing Seat Belt Use. PIN Flash 4 Background Data. ETSC, Brussels, 2007.

[15] Eurostat, 2009, http://epp.eurostat.ec.europa.eu/portal/page/portal/ transport/data/database.

[16] World Health Organization (WHO), The World Health Report 2006--Working together for Health. WHO, Geneva, 2006.

[17] E. Triantaphyllou, Multi-Criteria Decision Making Methods: A comparative Study, Kluwer Academic Publishers, Dordrecht, 2000. 